\title{
Backward masking and enhancement of multisegmented visual targets*
}

\author{
WILLIAM N. DEMBER, W. D. MATHEWS, \\ and MARY STEFL \\ University of Cincinnati, Cincinnati, Ohio 45221
}

The present experiment was designed to replicate and elaborate two earlier findings: (1) that certain visual targets comprising alternating black and white pie-shaped segments are both relatively difficult to detect in the absence of a masking figure and relatively easy to detect when followed by a masking stimulus, and (2) that detection of some such targets is actually enhanced when the annular masking figure is presented. In general, those results were obtained in this experiment, though enhancement was evident for targets having from 16 to 32 segments, whereas previous research suggested that the enhancement effect would fail somewhere between the values of 16 and 32 .

In two previous experiments (Dember \& Stefl, 1972; Ellis \& Dember, 1971), we have replicated and partially extended an interesting serendipitous finding, labeled backward enhancement, reported by Cox \& Dember (1970). ${ }^{1}$ Backward enhancement occurs in the paradigm which usually yields backward masking-i.e., the decreased detectability of a visual stimulus (the target) attributable to the presentation of a subsequent visual stimulus (the mask). In the case of backward enhancement, the masking stimulus serves to increase target detectability, but only for certain targets. Hopefully, if we can identify the properties of those targets which are subject to enhancement, we may be better able to infer the physiological mechanisms that underlie both masking and enhancement. The main purpose of the present experiment was to explore further one property of the targets used in our previous research that seems highly relevant-the number of pie-shaped black and white segments which the target disk comprises.

Our most recent experiment (Dember \& Stefl, 1972) employed target disks having $2,4,8,16$, and 32 segments as well as a homogeneous black disk. A clear-cut enhancement effect was obtained with the 16-segment target, consistent with earlier findings. There was somewhat ambiguous evidence for enhancement with the 8-segment target, but none for the other targets, which exhibited either little masking or the usual masking effect. In order better to specify the limits of the values at which enhancement occurs, the present experiment employed a homogeneous black disk and disks with $8,12,16,20,24,28$, or 32 black and white

*Supported by Grants EY 00481-06 from the National Eye Institute and GB 33621 from the National Science Foundation to William N. Dember. segments. We expected evidence for enhancement to be revealed somewhere between the values of 8 and 16 and to fail to appear between the values of 16 and 32 segments.

Though our main concern was with enhancement, we were also interested in replicating a trend, prominent in our earlier studies, for target detectability to decrease with increasing number of target segments in a nonmasking condition. The present experiment also allowed for a test of that finding.

\section{METHOD \\ Subjects}

Five female and three male college students with normal or corrected-to-normal vision served as paid Ss in the experiment. The Ss were trained for 3 days prior to the start of the experiment on each of the experimental conditions, for a total of 480 trials.

\section{Stimuli}

The eight target stimuli consisted of a homogeneous black disk and disks of $8,12,16,20,24,28$, or 32 alternating black and white segments, as shown in Fig. 1. The stimuli were constructed first of black cover paper on white bristol board. They were then photographed with Kodak High Contrast Copy film in a $35-\mathrm{mm}$ camera with normal lens such that the 10-in.-diam stimuli filled the frame. After the film was processed as prescribed by the manufacturer and contact printed on Agfa Brovira Grade 6 photographic paper, dulling spray was applied to reduce reflectance. The eight target stimuli were then mounted behind gray paper which reflected $50 \%$ of the light such that the diameter of the target disk was reduced to $8 \mathrm{~mm}$.

The masking stimulus comprised a pair of black rings horizontally arrayed on the same gray paper. The inner diameter of each ring was equal to the diameter of the target disk. The rings were $4 \mathrm{~mm}$ thick, and the distance between the centers of the rings was $18 \mathrm{~mm}$. The total display subtended $1 \mathrm{deg} 40 \mathrm{~min}$ of arc.

\section{Apparatus and Procedure}

Stimuli were presented tachistoscopically (Scientific Prototype Model GB). The apparatus was fitted with a metal plate that could be moved horizontally against adjustable stops so that the target stimuli could be centered in either of the two masking rings. The target disk could therefore easily be moved from one ring to the other. Viewing was monocular.

All eight Ss viewed each target stimulus presented alone (followed by a homogeneous flash of light) and with the masking rings. This yielded a total of 16 stimulus conditions.

The stimulus presentation was as follows: light fixation field, target, masking field, and light fixation field. Background illumination was kept constant at $10 \mathrm{fL}$ throughout. The duration of the masking flash was a constant $105 \mathrm{msec}$. Intertrial interval was approximately 10 sec.

During training, each $S$ received 30 practice trials on each condition. For half of the trials, target duration was $20 \mathrm{msec}$; for the other half, target duration was $10 \mathrm{msec}$. During both training and the experiment, the order of experimental conditions was randomized for each $\mathrm{S}$. Following the training days, each $\mathrm{S}$ experienced two experimental conditions on each of 8 days, with an approximate 10 -min break between conditions. 

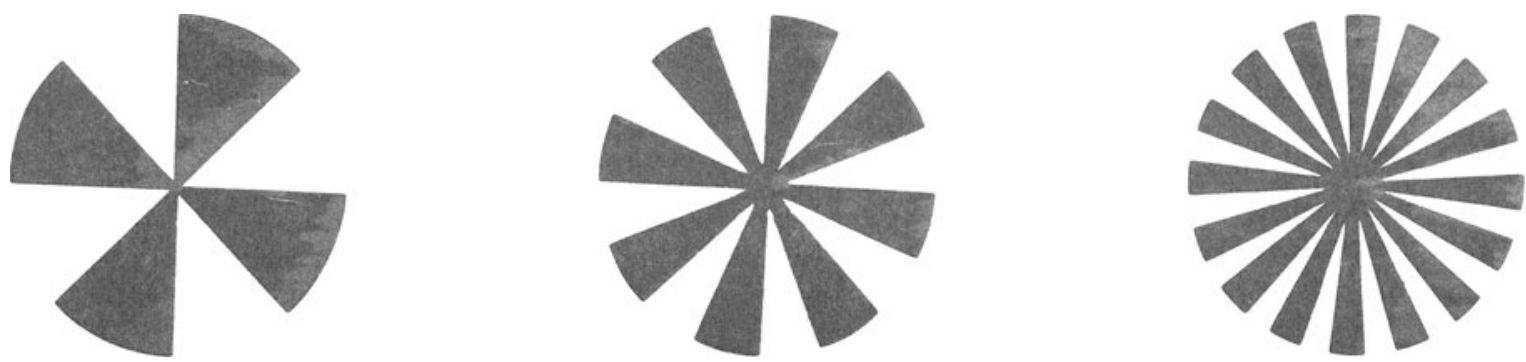

Fig. 1. Three of the target stimuli used in the present experiment, those, from left to right, with 8 , 16, and 32 segments.

Detection thresholds were determined for each condition according to the double-staircase variant of the method of limits (Dixon \& Massey, 1969). On a given trial, the E would position the target randomly in the center of one of the two rings (or on what would be the center if the rings were present in the case of the no-mask condition). Then, on a signal from $E$, the $S$ pressed a button activating the stimulus sequence and immediately responded with "left" or "right" to indicate target position, guessing when necessary. The stipulation was made that in order for a response to be counted correct, it must be made twice in a row. This reduced the probability of being correct by chance alone to 0.25 . Once a correct response was made, target duration was decreased; if the S's response was incorrect, target duration was increased. Whether the ascending or descending series of the staircase would be in effect on a given trial was randomized by $\mathrm{E}$ prior to the experimental session. Each block of 10 trials contained 5 trials from each series. Ten preliminary trials were conducted at the beginning of each experimental session, using 2-msec steps to determine the range in which the detection threshold was likely to fall. Thereafter, $0.5-\mathrm{msec}$ increments were used. The first 10 of the total 70 trials in the double-staircase procedure were not included in the computation of the detection threshold, but were designed to allow the two series to approach one another.

\section{RESULTS AND DISCUSSION}

The detection threshold for each $S$ in all conditions was computed using the procedure outlined by Dixon \& Massey (1969). These values were subjected to a two-way analysis of variance, repeated measures design. The ANOVA showed a highly significant target effect ( $F$ $=29.81, \mathrm{df}=7 / 49, \mathrm{p}<.001)$ and a highly significant interaction between target type and presence or absence of mask $(F=24.69, \mathrm{df}=7 / 49, \mathrm{p}<.001)$. Employing the Geiser-Greenhouse correction factor and conservative $\mathrm{df}$ changes the significance levels to .001 and .005 , respectively. The main effect of mask presence or absence was not significant.

The results are shown in Fig. 2, which plots mean detection threshold as a function of number of target segments for both the masking and nonmasking conditions. The effects apparent in Fig. 2 and confirmed in the analysis of variance replicate in general form those obtained in earlier studies: (1) target detectability under the nonmasking condition does decrease as the number of segments increases; (2) target enhancement does occur for certain multisegment targets.

The reliability of the enhancement effect for targets with 16, 20,24, 28, and 32 segments was assessed by simple $t$ tests, comparing the mean ratio of the masking

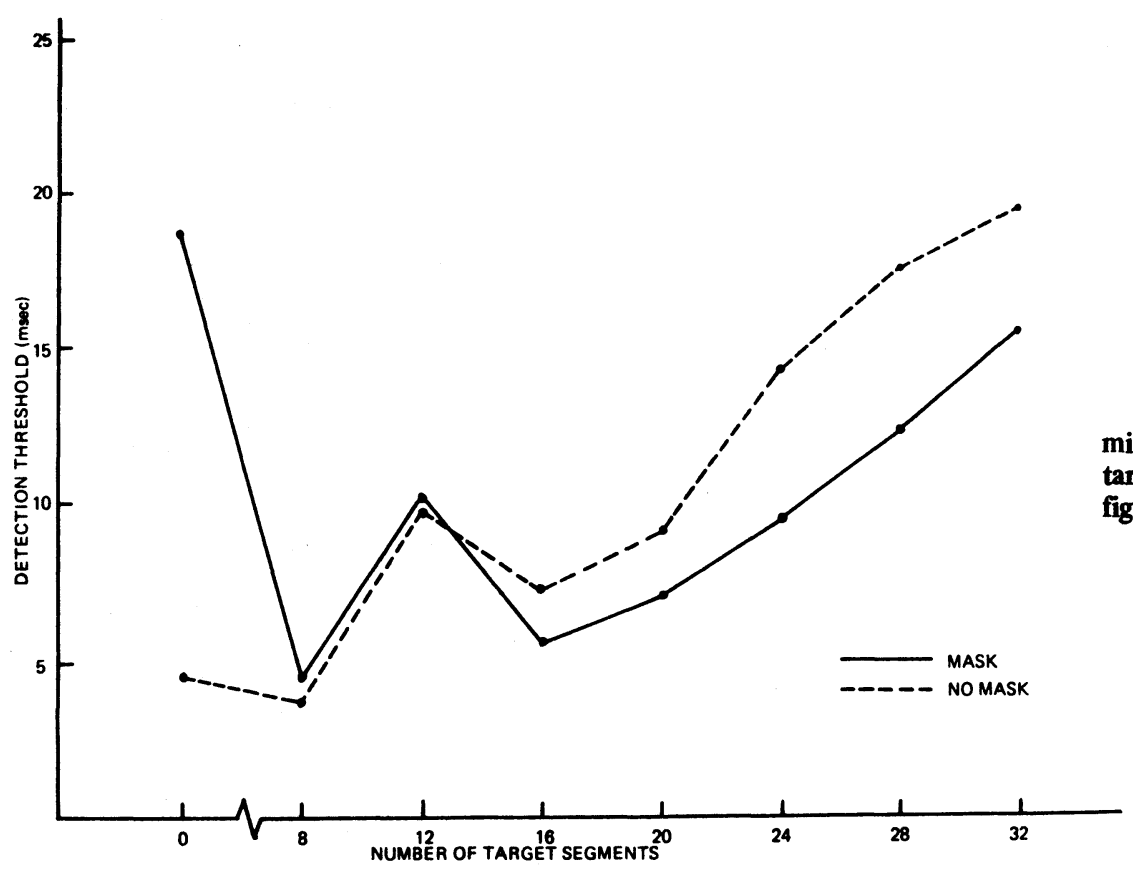

Fig. 2. Detection threshold in milliseconds as a function of number of target segments and presence of a masking figure. 
to nonmasking condition with a hypothetical ratio of 1.00. Enhancement would be indicated if the ratios had values significantly less than 1.00 . For all targets except the 20-segment one, a significant difference was found ( $p<.025$ or smaller). For the 20 -segmented target, $.05<\mathrm{p}<.10$. A similar analysis was done for the homogeneous disk and for the 8-and 12-segment targets to test for the conventional masking effect (as evidenced by mean ratios greater than 1.00 ). The three t values were, respectively, $7.24,1.53$, and .63 . Only the homogeneous disk showed significant masking $(p<.001)$. For the 8-segment target, $.05<p<.10$.

The present results differ somewhat in absolute terms from those reported by Dember \& Stefl (1972) in that the enhancement effect seems shifted to the right. That is, enhancement first becomes evident with the 16-segment target (and not with the 8 or 12) and remains evident at the 32-segment value (instead of dropping out entirely between 16 and 32 , as anticipated from the earlier results). We have no convincing explanation for this latter discrepancy except to note that the stimuli for the present experiment were prepared differently from those of our earlier studies. The use of the photographic technique employed in this experiment undoubtedly resulted in sharper contours and more uniform segments than were possible in making the stimuli used in the earlier studies, especially for the 32-segment target.

We obviously have not yet located an upper limit to the enhancement effect; further experiments using targets with more than 32 segments should be run to that end. In addition, it would be desirable to employ gratings varying in spatial frequency as targets. Use of gratings may enable us to relate our findings on detectability, maskability, and enhancement to the work of Davidson (1968) and others on what Cornsweet (1970) refers to as the spatial modulation transfer function. Using a contrast matching technique, Davidson (1968) found maximal apparent brightness of sine wave gratings to occur in a middle range of spatial frequencies. It is possible that our results reflect the operation of the same mechanism that mediates Davidson's, although Cornsweet $(1970$, p. 336) carefully points out that "threshold" measurements and brightness assessments are not necessarily equivalent, and of course our pie-shaped segments are quite different from gratings. In any event, further empirical work of the sort suggested should help narrow the range of potential mechanisms underlying masking and enhancement.

\section{REFERENCES}

Cornsweet, T. N. Visual perception. New York: Academic Press, 1970.

Cox, S. I., \& Dember, W. N. Backward masking of visual targets with internal contours. Psychonomic Science, 1970, 19, 255-256.

Davidson, M. L. Perturbation approach to spatial brightness interaction in human vision. Journal of the Optical Society of America, 1968, 58, 1300-1309.

Dember, W. N., \& Stefl, M. Backward enhancement? Science, $1972,175,93-95$.

Dixon, J. W., \& Massey, F. J. Introduction to statistical analysis. New York: McGraw-Hill, 1969.

Donchin, E., \& Lindsley, D. B. Retroactive brightness enhancement with brief paired flashes of light. Vision Research, 1965a, 5, 59-70.

Donchin, E., \& Lindsley, D. B. Visual evoked response correlates of perceptual masking and enhancement. Electroencephalography \& Clinical Neurophysiology, 1965b, 19, 325-335.

Ellis, D., \& Dember, W. N. Backward masking of visual targets with internal contours: A replication. Psychonomic Science, 1971, 22, 91-92.

\section{NOTE}

1. A similar effect, occurring under conditions quite different in several respects from those obtaining in our experiments, has previously been reported by Donchin \& Lindsley (1965a, b).

(Received for publication September 10, 1972.) 